\title{
Seizures due to multiple sclerosis: seven patients with MRI correlations
}

\author{
A J Thompson, A G Kermode, I F Moseley, D G MacManus, W I McDonald
}

\begin{abstract}
The MRI data of seven patients with clinically definite multiple sclerosis who developed epileptic seizures are presented. Six of these cases demonstrated new or enhancing lesions. Cerebral biopsy in one of these lesions confirmed inflammation with myelin breakdown products. Lesions implicated in the causation of seizures involved the cortex or subcortical area. In one patient, a new lesion was associated with EEG abnormalities which resolved as the lesion reduced in size. In three patients epileptic activity was the only clinical manifestation of disease. Large, unresolving lesions tended to be associated with continuing seizures.
\end{abstract}

$(\Im$ Neurol Neurosurg Psychiatry 1993;56:1317-1320)

The frequency of epilepsy in multiple sclerosis (MS) varies between $1 \%$ and $5 \%$ of patients ${ }^{1-5}$ which is considerably higher than the frequency in the normal population $(0.5-1 \%)$. Epilepsy is a common condition, however, and coincidental association must occur. A causative link is difficult to establish as many of the earlier studies do not mention the diagnostic criteria used to classify the affected patients, nor do they give a description of the fits. There has also been considerable confusion between epilepsy and paroxysmal tonic seizures. The latter are not truly epileptic as they arise from the spinal cord or brain stem and are due to axonal rather than neuronal dysfunction. ${ }^{67}$ Despite these reservations there does appear to be a small group of patients in which a causal association between epileptic seizures and MS is likely, and it has been suggested that it may relate to cortical or subcortical involvement, a not infrequent finding at postmortem examination. ${ }^{8}$

The sensitivity of MRI in detecting the white matter lesions of MS is now well established. ${ }^{9}$ Involvement of the grey matter is less often seen, though this may be in part due to the fact that the $T_{1}$ and $T_{2}$ of MS lesions are similar to those of grey matter so that cortical or subcortical lesions may be indistinguishable from normal grey matter. ${ }^{10}$ The use of the contrast agent gadolinium-DTPA (GdDTPA) in acute lesions helps to overcome this difficulty. ${ }^{10}$
The MRI data of seven patients with MS who developed epileptic seizures in the course of their illness is presented.

\section{Methods}

All patients had clinically definite $\mathrm{MS},{ }^{11}$ and had been referred to the National Hospital for Neurology and Neurosurgery, Queen Square, during a two-year period. All had oligoclonal bands in their CSF and other diseases such as systemic lupus erythematosus had been excluded.

$\mathrm{T}_{2}$-weighted MRI of the brain was performed on a Picker 0.5 T super-conducting machine (SE2000/60, 5-mm contiguous axial slices). When Gd-DTPA was used as a contrast agent $\mathrm{SE500/40} \quad\left(\mathrm{T}_{1}\right.$-weighted $)$ sequences before and after contrast were obtained. The second MRI on patient 1 was obtained on a $1.5 \mathrm{~T}$ system (SE3000/80).

\section{Results}

The seven patients were aged between 34 and 59 years (mean 42 years). There were three women and four men. Disease duration before the onset of the fits varied between three and 20 years (mean nine years). Disability varied between 1.0 and $7 \cdot 0$ (mean 3.0) on Kurtzke's Expanded Disability Status Scale $^{12}$ (EDSS). The table summarises the chemical, EEG and MRI data.

\section{Case 1}

A 59-year-old female with a five-year history of MS developed vertigo with weakness of the left arm and leg. Two days later she had a generalised tonic-clonic seizure which began in the left leg. She was disorientated and on examination had nystagmus on right lateral gaze, ataxia, bilateral limb weakness, and extensor plantar responses. An MRI scan was performed on the day of the seizure and, when compared with a study performed 18 weeks earlier, there were marked changes: a number of pre-existing lesions had enlarged and there were multiple new lesions some of which extended to the cortex (fig 1). Contrast enhanced CT showed enhancement in the right parietal lesion indicating recent activity at this site. ${ }^{13}$ An EEG was not done and the patient was not on anticonvulsant medication. Despite having a number of relapses over the subsequent four years she had no further seizures. 
Table Clinical, EEG and MRI activity of seven patients with multiple sclerosis with epilepsy of recent onset

\begin{tabular}{|c|c|c|c|c|c|c|c|}
\hline Patient & $\begin{array}{l}\text { Seizures } \\
\text { associated with } \\
\text { clinical activity }\end{array}$ & Abnormal EEG & $\begin{array}{l}\text { Recent activity } \\
C T / M R I\end{array}$ & $\begin{array}{l}\text { Cortical/ } \\
\text { subcortical } \\
\text { involvement }\end{array}$ & $\begin{array}{l}\text { Correlation } \\
\text { between } M R I \\
\text { and } E E G\end{array}$ & $R_{\mathrm{x}}$ & $\begin{array}{l}\text { Continued } \\
\text { fits }\end{array}$ \\
\hline 1 & relapse & not done & $\begin{array}{l}\text { enhancing right } \\
\text { parietal lesion }\end{array}$ & yes & - & no & no \\
\hline 2 & no & $\begin{array}{l}\text { spike \& wave left } \\
\text { anterior temporal }\end{array}$ & $\begin{array}{l}\text { possible left } \\
\text { temporal }\end{array}$ & yes & yes & yes & no \\
\hline 3 & $\begin{array}{l}\text { relapse } \\
5 / 52 \text { later }\end{array}$ & $\begin{array}{l}\text { left } \\
\text { posterior parietal } \\
\text { spike \& wave }\end{array}$ & $\begin{array}{l}\text { possible new } \\
\text { lesion } \\
\text { posterior parietal }\end{array}$ & yes & yes & yes & no \\
\hline 4 & no & $\begin{array}{l}\text { bitemporal } \\
\text { spike \& wave }\end{array}$ & $\begin{array}{l}\text { new lesion } \\
\text { right trigone }\end{array}$ & yes & yes & no & no \\
\hline 5 & & diffuse slowing & left frontal & yes & & no & no \\
\hline 6 & $\begin{array}{l}\text { cognitive } \\
\text { deterioration }\end{array}$ & left sided slowing & left frontal & yes & possible & yes & yes \\
\hline 7 & $\begin{array}{l}\text { cognitive } \\
\text { deterioration }\end{array}$ & diffuse slowing & left parietal & yes & no & yes & yes \\
\hline
\end{tabular}

Case 2

A 37-year-old male had his first generalised seizure eight years after the diagnosis of MS. Three years later he experienced two further seizures and three months following that he had an episode of status epilepticus. An EEG showed repeated epileptiform discharges in the left anterior temporal region which increased markedly on hyperventilation. Examination revealed bilateral internuclear ophthalmoplegia with mild spastic quadriparesis and ataxia (EDSS $=3 \cdot 0$ ). MRI was carried out within a week of the episode of status epilepticus and showed numerous lesions in the brain stem, cerebellum, periventricular white matter, and corpus callosum. There was a small subcortical lesion in the left temporal lobe. The patient was commenced on carbamazepine. No further seizures occurred in the following two years.

Case 3

A 41-year-old female with minimal disability (EDSS $=1.5)$ and a 20-year history of MS experienced a generalised seizure which was followed by four further fits over 14 days. She had no other new symptoms or signs. An

Figure $1 \quad T_{2}$-weighted $\operatorname{MRI}\left(S E_{2000160}, 0.5 \mathrm{~T}\right)$ of patient 1 showing a large paramedian right parietal lesion involving the cortex.

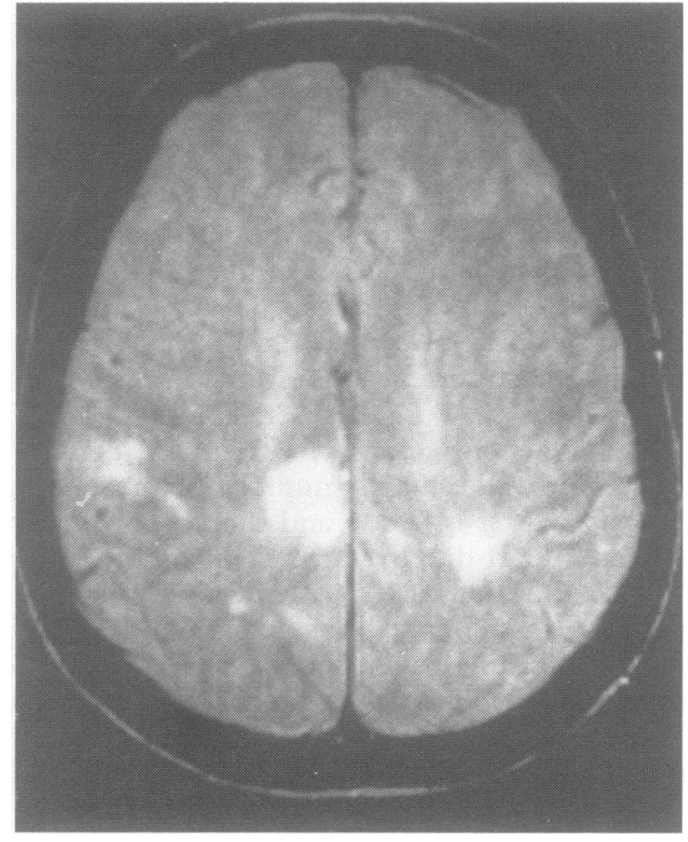

EEG showed frequent spike and sharp wave discharges over the left parietal region with some generalised slowing. She was commenced on sodium valproate. Five weeks later she had a mild cord relapse consisting of sensory loss below the waist. This was associated with a further fit which was preceded by an aura consisting of a strange, awful sensation in the mouth. An MRI showed numerous periventricular lesions with several small subcortical lesions in the left posterior parietal region. She continues on anticonvulsant medication and has had no further fits after two years.

\section{Case 4}

This 34-year-old patient with a four-year history of MS and minimal disability (EDSS = 1) had a generalised tonic-clonic seizure followed over the next few days by a number of complex partial seizures. An EEG done on the first day showed spike and wave discharge in the right temporal region, with bitemporal slowing more marked on the right. An MRI showed a number of new subcortical lesions in the right parietal lobe and a large lesion posterior to the right trigone. In the next two weeks the patient had a number of minor seizures lasting a few minutes during which he would feel light headed and out of touch. He decided against any anticonvulsant medication and repeat MRI showed marked reduction in the size of the lesion in the right trigone. A repeat EEG five months later was completely normal and he has had no further seizures over the two year follow up.

Case 5

A 34-year-old female with a four-year history of MS experienced a generalised tonic-clonic seizure. On examination she had pseudobulbar speech and weakness in all four limbs, most marked in the left leg, with bilateral extensor plantars (EDSS $=5$ ). EEG showed diffuse slowing with no focal abnormality. An MRI performed seven days after this seizure showed two large enhancing frontal lesions on the left and right, known to be new from serial scans. The following day the patient had another generalised seizure. She was not given any anticonvulsant medication and had no further episodes over the subsequent two years. 
Figure $2 \quad T_{2}$-weighted $M R I\left(S E_{2000600} 0.5 T\right)$ of patient 6 showing a large right frontal lesion with mass effect. High signal extends to the surface of the brain.

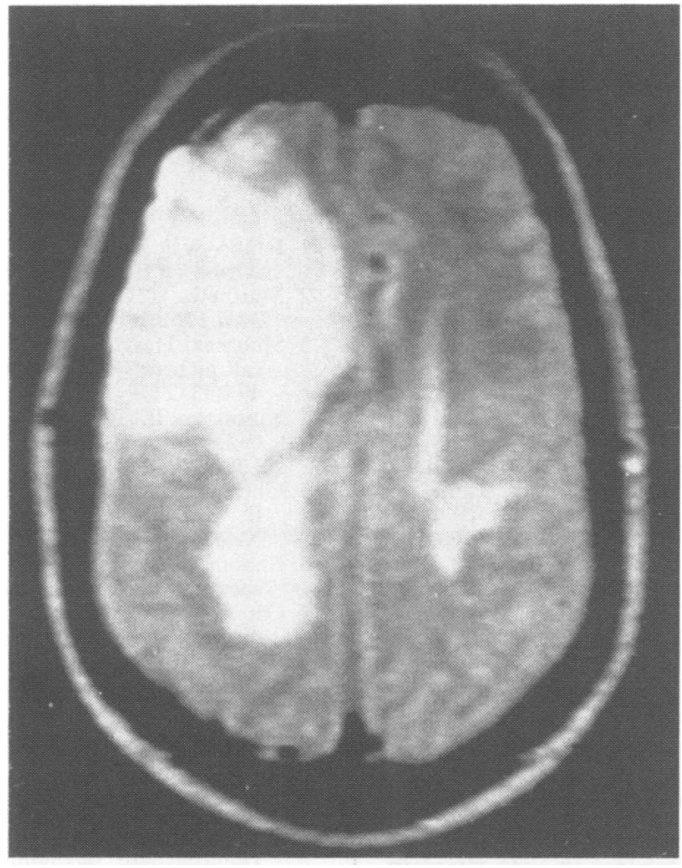

Case 6

A 53-year-old male with a six-year history of MS presented in a confusional state following two months progressive deterioration in cognitive function. He had bilateral limb ataxia and spasticity and was unable to transfer from bed to chair (EDSS = 8). CT showed an extensive right frontal area of mixed but mainly low attenuation tissue causing mass effect. It showed patchy peripheral and central enhancement. A large frontal lesion with extensive cortical involvement was seen on MRI (fig 2). A right posterior parietal lesion not seen on CT also involved the cortex. Multiple other areas of white matter high signal were also seen. As the patient was being removed from the scanner he had a generalised tonic-clonic seizure and he had a number of further seizures over the next four days. EEG demonstrated generalised slowing but no epileptiform activity. A provisional diagnosis of malignant astrocytoma was made and he underwent biopsy. At surgery the brain appeared extremely oedematous and histology showed features of subacute demyelination. $\mathrm{He}$ has continued to deteriorate with further fits despite medication. Other aspects of this case have been reported elsewhere. ${ }^{14}$

Case 7

A 37-year-old male with a nine-year history of MS had a series of generalised tonic-clonic seizures associated with a progressive dominant hemisphere syndrome characterised by dysphasia and dysgraphia. Examination revealed an incomplete right homonymous hemianopia in addition to bilateral pyramidal and cerebellar signs which were known to be of long standing. CT showed a low density lesion in the left temporoparietal region with ring enhancement but no mass effect. MRI showed extensive white matter lesions predominantly involving the left hemisphere.
There was extensive confluent high signal in the left parietal, temporal and posterior frontal lobes with extensive subcortical involvement. EEG showed severe slowing, particularly over the left temporoparietal area. Repeat MRI four months later was unchanged. His seizures have continued at a rate of three per year despite treatment with carbamazepine. Other aspects of this case have been reported elsewhere. ${ }^{15}$

\section{Discussion}

All seven patients had generalised seizures. In one the onset was focal and another had complex partial seizures. Clinically there was a wide variation in age, disease duration, and disability between patients. All EEG recordings were abnormal with three showing spike and wave discharges and three showing slowing which in one patient (patient 6) was asymmetrical. There was a correlation between the site of EEG abnormality and MRI activity in patients 2 and 3 and to a lesser extent in patients 4 and 6.

Seizures were associated with disease activity in six patients. In four this was clinically manifest (patients 1, 3,6 and 7) whereas in two, new MRI activity was seen in the absence of clinical signs (patients 4 and 5). In patient 3 the clinical activity was in the spinal cord five weeks after the first seizure with no symptoms relevant to the new cerebral lesions seen on MRI. In three of the seven patients, epilepsy was therefore the only clinical manifestation of the new active lesion. It is now well established using $M R I^{16}$ that clinically silent lesions are common in MS and it is not surprising that a seizure may be the only manifestation of a new lesion. A similar finding is well documented in CT studies of patients with cerebrovascular disease. ${ }^{17}$

Given that the occurrence of seizures is frequently associated with new MRI activity, the significance of the site and size of the new lesions may be usefully addressed. Two patients had cortical lesions and in the other five there was clear subcortical involvement. Two patients had very large lesions, whereas in the remaining five they were small. The former were associated with progressive cognitive deficit and continuing seizures reminiscent of the three cases presented by Sagar et $a l,{ }^{18}$ in whom seizures were associated with large lesions which clinically and radiologically mimicked tumours. It would appear therefore that seizures in MS result from new lesions which may be either cortical or subcortical in location. A similar observation with regard to site has been made in relation to epilepsy associated with cerebrovascular disease, ${ }^{19}$ though a more recent study has suggested that lacunar infarction without obvious cortical or subcortical involvement may also cause late onset epilepsy. ${ }^{17}$

The risk of further seizures is a more difficult issue. Matthews ${ }^{20}$ suggests patients divide into three groups: (a) those in whom seizures were associated with clinical relapse-these patients rarely had further seizures; (b) those 
in whom seizures did not relate to clinical activity-these patients tended to have occasional seizures though this was not invariable and control was relatively straightforward; and (c) those in whom seizures were associated with progressive cognitive decline - these patients had a poor prognosis and were susceptible to status epilepticus. In the present study patients $1-5$ would fit well into group (a), although without MRI, disease activity would not have been detected in them all. All five patients were seizure free during the follow up period of two years. Patients 6 and 7, who had large unresolving lesions, fall between groups (b) and (c) and have continued to have seizures despite medication.

Does the association of seizures with new evolving lesions help explain their mechanism? Serial MRI studies have shown that in MS, new lesions increase in size over a 4-6 week period ${ }^{1016}$ and then reduce slowly. This time scale, together with evidence from $T_{2}$ magnetisation decay curves, suggests that oedema is largely responsible for this fluctuation in size. Thus it would be reasonable to suggest that the development of oedema in critically located lesions may play a part in seizure production. This would be supported by patient 4 in whom EEG and clinical activity resolved as the subcortical lesion reduced in size. It would also explain the good prognosis in this group in relation to further fits. In patients with large, unchanging cortical lesions it would be reasonable to suggest that the plaque itself is acting as an epileptic focus and that these patients may have a continuing tendency to seizure activity. These mechanisms would parallel those suggested in cerebrovascular disease in which most seizures occur during the acute phase and may result from hyperaemia producing transient, cytotoxic, metabolic alterations. ${ }^{21}$ In chronic cases seizures may occur when there is structural damage which may in itself be epileptogenic.

In conclusion the results from this small group of patients support the suggestion that there is a causal connection between MS and epilepsy and suggests that these seizures result from cortical or subcortical lesions.
The NMR Research Group is supported by the Multiple Sclerosis Society of Great Britain and Northern Ireland, and Sclerosis Society of Great Britain and Northern Ireland, and
also by the Medical Research Council of the United King by

The contribution of Dr Simon D Shorvon, Institute of The contribution of Dr Simon D Shorvon, Institute of
Neurology, Queen Square, London is gratefully acknowledged.

1 Drake WE, Macrae D. Epilepsy in multiple sclerosis. Neurology 1961;11:810-6.

2 Matthews WB. Epilepsy and disseminated sclerosis. $Q \mathcal{F}$ Med 1962;1:141-55.

3 Shibasaki H, McDonald WI. Racial modification of clinical picture of multiple sclerosis. $\mathcal{f}$ Neurol Sci 1981 , 49:253-71.

4 Kinnunen E, Wikstrom J. Prevalence and prognosis of epilepsy in patients with multiple sclerosis. Epilepsia 1986;27:729-33.

5 Ghezzi A, Montanini R, Basso PF, Zaffaroni M, Massimo E, Cazzullo CL. Epilepsy in multiple sclerosis. Eur Neurol 1990;20:218-23.

6 Ekbom KA, Westerberg C-E, Osterman PO. Focal sensory-motor seizures of spinal origin. Lancet 1968;1:67.

7 Osterman PO, Westerberg C-E. Paroxysmal attacks in multiple sclerosis. Brain 1975;98:189-202.

8 Brownell B, Hughes JT. The distribution of plaques in the cerebrum in multiple sclerosis. $f$ Neurol Neurosurg cerebrum in multiple sclerc

9 Ormerod IEC, Miller DH, McDonald WI, et al. The role of NMR imaging in the assessment of multiple sclerosis and isolated neurological lesions: a quantitative study. Brain 1987;110:1579-616.

10 Kermode AG, Tofts PS, Thompson AJ, et al. Heterogeneity of blood-brain barrier changes in multiple sclerosis: an MRI study with gadolinium-DTPA enhancement. Neurology 1990;40:229-35.

11 Poser CM, Paty DW, Scheinberg L, et al. New diagnostic criteria for multiple sclerosis: guidelines for research protocols. Ann Neurol 1983;13:227-31.

12 Kurtzk JF. Rating neurologic impairment in multiple sclerosis: an expanded disability status scale (EDSS). Neurology 1983;33:1444-52.

13 Kermode AG, Thompson AJ, Tofts PS, et al. Breakdown of the blood-brain barrier precedes other MRI signs of new lesions in multiple sclerosis: pathogenetic and clininew lesions in multiple sclerosis: pathogen
cal implications. Brain 1990;113:1477-89.

14 Youl BD, Kermode AG, Thompson AJ, et al. Destructive lesions in demyelinating disease. $\mathcal{F}$ Neurol Neurosurg Psychiatry 1991;54:288-92.

15 Plant GT, Kermode AG, Turano G, et al. Symptomatic retrochiasmal lesions in multiple sclerosis: clinical features, visual evoked potentials and magnetic resonance imaging. Neurology 1992;42:68-76.

16 Thompson AJ, Kermode AG, MacManus DG, et al. Major differences in the dynamics of primary and secondary progressive multiple sclerosis. Ann Neurol 1991;29:53-62.

17 Roberts RC, Shorvon SD, Cox TCS, Gilliatt RW. Clinically unsuspected cerebral infarction revealed by computed tomography scanning in late onset epilepsy. computed tomography scan
Epilepsia 1988;29:190-4.

18 Sagar HJ, Warlow CP, Sheldon PWE, Esiri MM Multiple sclerosis with clinical and radiological features of cerebral tumour. $\mathcal{F}$ Neurol Neurosurg Psychiatry 1982; 45:802-8.

19 Richardson EP, Dodge PR. Epilepsy in cerebral vascular disease. Epilepsia 1954;3:49-65.

20 Matthews WB. Symptoms and signs. In: Matthews WB edn. McAlpine's Multiple Sclerosis 2nd edn. Edinburgh: Churchill Livingstone, 1991:61-3.

21 Kieburtz K, Ricotta J, Moxley RT. Seizures following carotid endarterectomy. Arch Neurol 1990;47:568-70. 